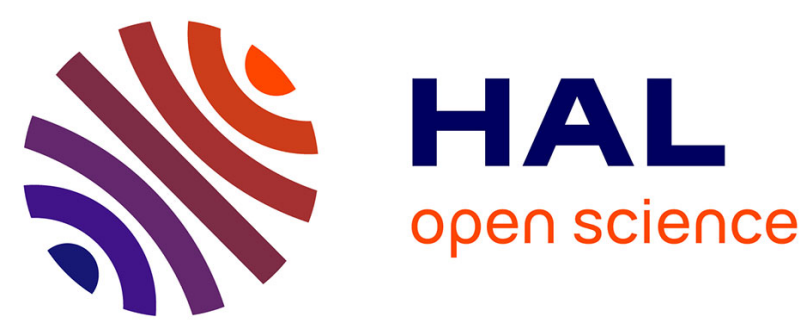

\title{
Observer design for a class of parabolic systems with large delays and sampled measurements
}

Tarek Ahmed-Ali, Emilia Fridman, Fouad Giri, Mohamed Kahelras, Françoise Lamnabhi-Lagarrigue, Laurent Burlion

\section{- To cite this version:}

Tarek Ahmed-Ali, Emilia Fridman, Fouad Giri, Mohamed Kahelras, Françoise Lamnabhi-Lagarrigue, et al.. Observer design for a class of parabolic systems with large delays and sampled measurements. IEEE Transactions on Automatic Control, 2019, pp.1-1. 10.1109/TAC.2019.2941434 . hal-02409383

\section{HAL Id: hal-02409383 \\ https://hal-centralesupelec.archives-ouvertes.fr/hal-02409383}

Submitted on 13 Jul 2020

HAL is a multi-disciplinary open access archive for the deposit and dissemination of scientific research documents, whether they are published or not. The documents may come from teaching and research institutions in France or abroad, or from public or private research centers.
L'archive ouverte pluridisciplinaire HAL, est destinée au dépôt et à la diffusion de documents scientifiques de niveau recherche, publiés ou non, émanant des établissements d'enseignement et de recherche français ou étrangers, des laboratoires publics ou privés. 


\title{
Observer design for a class of parabolic systems with large delays and sampled measurements.
}

\author{
Tarek Ahmed-Ali ${ }^{1}$, Emilia Fridman ${ }^{2}$, Fouad Giri ${ }^{1}$, Mohamed Kahelras ${ }^{3}$, Francoise Lamnabhi-Lagarrigue ${ }^{3}$ \\ Laurent Burlion ${ }^{4}$
}

\begin{abstract}
In this paper, we design a novel observer for a class of semilinear heat 1D equations under the delayed and sampled point measurements. The main novelty is that the delay is arbitrary. To handle any arbitrary delay, the observer is constituted of a chain of sub-observers. Each sub-observer handles a fraction of the considered delay. The resulting estimation error system is shown to be exponentially stable under a sufficient number of sub-observers is used. The stability analysis is based on a specific Lyapunov-Krasovskii functional and the stability conditions are expressed in terms of LMIs.
\end{abstract}

\section{INTRODUCTION}

This paper deals with the design of observers for a class of parabolic PDEs with delayed and sampled measurements. This problem is highly challenging since time delays affecting output measurements appears in many applications. We can cite the well-known networked control systems (NCSs) which are systems controlled and supervised by remote controllers and observers through a communication device. The main works existing in the literature are focused on finite-dimensional systems described by ODEs, see e.g. cite [1] and reference list therein. The main idea consisted in a re-design of an existing exponentially convergent state observer for the delay-free system so that exponential convergence is preserved in the presence of time-delay. The modification essentially consists in introducing of state predictors to compensate for time delay. This has been illustrated with several classes of observers based on drift-observability property [2] or on high-gain observers [3], [1] , [4] . However, for nonlinear ODEs, the designed predictor is useful in compensating the delay effect only up to some upper limit. Then to enlarge the maximum time-delay, a chain of predictors simultaneously operating are introduced [2].

In parallel with the above "finite-dimensional" research activity, the "infinite-dimensional" backstepping transformation for linear systems based approach has first been developed, see e.g. [5] and references therein. This approach consists in letting the output sensor delay be

\footnotetext{
*This work was not supported by any organization

${ }^{1}$ Tarek Ahmed-Ali and Fouad Giri are with Laboratoire d'Automatique de Caen, Universit de Caen Normandie, ENSICAEN,14000 Caen, France, ahmed_alifr@yahoo.fr, fouadgiridyahoo.fr

${ }^{2}$ Emilia Fridman with the School of Electrical Engineering, Tel-Aviv University, Tel-Aviv 69978, Israel emilia@eng.tau.ac.il

${ }^{3}$ Mohamed Kahelras and Francoise Lamnabhi-Lagarrigue are with Laboratoire des Signaux et Systmes (L2S), CNRS-CentraleSuplec-Université Paris Sud, Université Paris Saclay, 91192 Gif-sur-Yvette, France, mohamed.kahelrasal2s.centralesupelec.fr, Francoise. Lamnabhi-Lagarrigued12s. centralesupelec.fr

${ }^{4}$ Laurent Burlion , ONERA, 31000 Toulouse, Francelaurent. Burliondonera.fr
}

captured by a first-order hyperbolic PDE. Then, full-order observers are designed that estimate both the system (finite-dimensional) state and the sensor (infinite-dimensional) state. The extension of this approach to (triangular) nonlinear systems has been studied in [6], where a high-gain type observer has been developed. The arbitrary-size delay effect has been compensated for by developing a PDE version of the chain observer concept.

The problem of observer design for nonlinear PDEs with arbitrary delays measurements has yet to be solved. In this paper, the problem is addressed for a class of parabolic PDEs under point measurements as in [7]. In the latter paper the results were ined to small delays. To compensate the effect of the arbitrary-size delay, the concept of chain-observer is extended to fit this class of systems. Accordingly, the initial delay PDE system representation is re-expressed in the form of fictive delayed subsystems. The observer is composed of elementary observers connected in series. The interconnection is such that the first elementary observer is directly driven by the physical system output. Then, the elementary observer is driven by a virtual output generated by the previous observer. Each elementary observer can be viewed as a predictor which compensates for the effects of the fractional time-delay. As in [7], using an appropriate Lyapunov-Krasovskii functional, sufficient conditions are established in terms of LMIs via Halanay's inequality [14].

Note that in the existing results [3], [6], the exponential convergence can be proved by induction via input-to-state stability of the sub-observers and by employing the fact that the input is exponentially converging. This approach is not applicable here because of Halanay's inequality that has been extended to the case of uniformly bounded inputs only (see Lemma 1 of [13]). The latter may lead to a practical stability. Here we suggest a novel proof which employs a special construction of a Lyapunov-Krasovskii functional for the augmented system of sub-observers. The sufficient conditions involve a sufficient number of elementary observers: the larger the delay the larger the number of observers. Extension to sampled data delayed measurements is presented.

It has to be noticed that a conference version of the present work will be presented at [16]. The main differences between the present work and [16] are in the novel proof of Theorem 1 and in the more detailed proof of Theorem 2. We also add new simulations to more highlight the behavior of our algorithm and show the effect of the number of observers depending on the delay value. The paper is organized as follows: first, the observation problem under study is formulated in Section 
2; then, the observer design with delayed measurements and analysis are dealt within Sections 3; In section 4, the extension to sampled-data case is presented. In section 5, we illustrate our results by some simulations on a numerical example involving both delays and sampling measurements.

\section{Notations and preliminaries}

Throughout the paper the superscript $T$ stands for matrix transposition, $\mathbf{R}^{n}$ denotes the $\mathrm{n}$-dimensional Euclidean space with vector norm |.|, $\mathbf{R}^{n \times m}$ is the set of all $n \times m$ real matrices, and the notation $P>0$, for $P \in \mathbf{R}^{n \times n}$, means that $P$ is symmetric and positive definite. In matrices, symmetric terms are denoted $* ; \lambda_{\min }(P)\left(\right.$ resp. $\left.\lambda_{\max }(P)\right)$ denotes the smallest (resp. largest) eigenvalue. The notation $\left(t_{i}\right)_{i \geq 0}$ refers to a strictly increasing sequence such that $t_{0}=0$ and $\lim t_{i}=\infty$. The sampling periods are bounded i.e. $0<t_{i+1}^{k \rightarrow \infty}-t_{i}<h$ for some scalar $0<h<\infty$ and all $i=0,1, \ldots, \infty$. We also define the variable $\tau(t)=t-t_{i}, t \in\left[t_{i}, t_{i+1}\right) . L_{2}(0, l)$ is the Hilbert space of square integrable functions $z(x), x \in[0, l]$ with the corresponding norm $\|z(x)\|_{L_{2}}=\sqrt{\int_{0}^{l} z^{2}(x) d x} . \mathcal{H}^{1}(0, l)$ is the Sobolev space of absolutely continuous functions $z:(0, l) \rightarrow \mathbf{R}$ with the square integrable derivative $\frac{d}{d x}$. $\mathcal{H}^{2}(0, l)$ is the Sobolev space of absolutely continuous functions $\frac{d z}{d x}:(0, l) \rightarrow \mathbf{R}$ and with $\frac{d^{2} w}{d x^{2}} \in L_{2}(0, l)$. Given a two-argument function $u(x, t)$, its partial derivatives are denoted $u_{t}=\frac{\partial u}{\partial t}, u_{x x}=\frac{\partial^{2} u}{\partial x^{2}}$. Throughout the paper the following lemma will be used to prove exponential convergence of our observer.

Lemma 1: ( Halanay's type Inequalities [9])

Let $0<\delta_{1}<2 \delta$ and let $V:\left[t_{0}-h, \infty\right) \rightarrow[0, \infty)$ be an absolutely continuous function which satisfies

$$
\dot{V}(t) \leq-2 \delta V(t)+\delta_{1} \sup _{-h \leq s \leq 0} V(t+s)
$$

Then

$$
V(t) \leq e^{-2 \alpha\left(t-t_{0}\right)} \sup _{-h \leq s \leq 0} V(t+s)
$$

where $\alpha$ is the unique positive solution of the equation

$$
\alpha=\delta-\frac{\delta_{1} e^{2 \alpha h}}{2}
$$

\section{SYSTEM DESCRIPTION}

We consider a semi-linear diffusion equation:

$$
u_{t}(x, t)=u_{x x}(x, t)+f(u(x, t), x, t)
$$

with Dirichlet conditions $u(0,0)=u(l, 0)=0$. The system output is, $y(t)=u\left(\bar{x}_{j}, t-D\right)$ where $\bar{x}_{j}=\frac{x_{j+1}+x_{j}}{2}(j=$ $0, \ldots, N-1)$ and the points $x_{j}$ divide the interval $[0, l]$ such that $0=x_{0}<\ldots<x_{N}=l$. It is supposed that $x_{j+1}-x_{j} \leq$ $\Delta$. The constant $D$ represents an arbitrarily delay and $N$ is the number of distributed sensors. It is also supposed that the function $f$ is known, of class $\mathcal{C}^{1}$, and satisfying $m_{f} \leq f_{u} \leq$ $M_{f}$, for some scalar constants $m_{f}$ and $M_{f}$.

\section{OBSERVER DESIGN}

We will present an observer, constituted by a chain of $m$ sub-observers, which ensures exponential convergence for an arbitrarily delay $D$. Each sub-observer estimates the state $u\left(x, t+\frac{k}{m} D-D\right)$ by using the estimation provided by the previous one in the chain whereas the first sub-observer uses the delayed measurement provided by sensors. The last sub-observer in the chain provides the estimation of the $u(x, t)$. As we will see below, by using a suitable Lyapunov functional, we will derive sufficient conditions involving both delay $D$, and the number of sub-observers in the chain $m$.

As in [2] we introduce the following notations for the delayed states :

$$
\begin{gathered}
u^{0}(x, t)=u(x, t-D), \\
u^{k}(x, t)=u\left(x, t+\frac{k}{m} D-D\right), \quad k=1 \ldots, m
\end{gathered}
$$

Using these notations we easily check that :

$$
u^{k+1}(x, t)=u^{k}\left(x, t-\frac{D}{m}\right)
$$

and

$$
u^{m}(x, t)=u(x, t)
$$

where $m$ is the number of sub-observers in the considered chain.

We propose the following observer structure :

for $k=1$ :

$$
\begin{aligned}
\hat{u}_{t}^{1}(x, t)= & \hat{u}_{x x}^{1}(x, t)+f\left(\hat{u}^{1}(x, t), x, t\right) \\
- & L\left(\hat{u}^{1}\left(\bar{x}_{j}, t-\frac{D}{m}\right)-y(t)\right), \\
& \forall x \in\left[x_{j}, x_{j+1}\right),
\end{aligned}
$$

for $k=2, \ldots, m$ :

$$
\begin{aligned}
\hat{u}_{t}^{k}(x, t)= & \hat{u}_{x x}^{k}(x, t)+f\left(\hat{u}^{k}(x, t), x, t\right) \\
- & L\left(\hat{u}^{k}\left(\bar{x}_{j}, t-\frac{D}{m}\right)-\hat{u}^{k-1}\left(\bar{x}_{j}, t\right)\right), \\
& \forall x \in\left[x_{j}, x_{j+1}\right),
\end{aligned}
$$

It is readily checked that the observation error systems $e^{k}(x, t)=\hat{u}^{k}(x, t)-u^{k}(x, t)$ undergoes the following equations:

for $k=1$ :

$$
\begin{aligned}
e_{t}^{1}(x, t)= & e_{x x}^{1}(x, t)+f\left(\hat{u}^{1}(x, t), x, t\right)-f\left(u^{1}(x, t), x, t\right) \\
- & L e^{1}\left(\bar{x}_{j}, t-\frac{D}{m}\right), \\
& \forall x \in\left[x_{j}, x_{j+1}\right),
\end{aligned}
$$

for $k=2, \ldots, m$ :

$$
\begin{aligned}
e_{t}^{k}(x, t)= & e_{x x}^{k}(x, t)+f\left(\hat{u}^{k}(x, t), x, t\right)-f\left(u^{k}(x, t), x, t\right) \\
- & L\left(\hat{u}^{k}\left(\bar{x}_{j}, t-\frac{D}{m}\right)-\hat{u}^{k-1}\left(\bar{x}_{j}, t\right)\right), \\
& \forall x \in\left[x_{j}, x_{j+1}\right),
\end{aligned}
$$


Note that

$$
u^{k}\left(x, t-\frac{D}{m}\right)=u^{k-1}(x, t)
$$

Then, for $k=1$ :

$$
\begin{aligned}
e_{t}^{1}(x, t)= & e_{x x}^{1}(x, t)+\phi\left(x, t, e^{1}\right)\left(\hat{u}^{1}(x, t)-u^{1}(x, t)\right) \\
- & L e^{1}\left(\bar{x}_{j}, t-\frac{D}{m}\right), \\
& x \in\left[x_{j}, x_{j+1}\right), \\
e^{1}(l, t)= & e^{1}(0, t)=0,
\end{aligned}
$$

for $k=2, \ldots, m$ :

$$
\begin{aligned}
e_{t}^{k}(x, t)= & e_{x x}^{k}(x, t)+\phi\left(x, t, e^{k}\right)\left(\hat{u}^{k}(x, t)-u^{k}(x, t)\right) \\
- & L e^{k}\left(\bar{x}_{j}, t-\frac{D}{m}\right)+L e^{k-1}\left(\bar{x}_{j}, t\right), \\
& \forall x \in\left[x_{j}, x_{j+1}\right), \\
e^{k}(l, t)= & e^{k}(0, t)=0,
\end{aligned}
$$

where

$$
\phi\left(x, t, e^{k}\right)=\int_{0}^{1} f_{u}\left(\hat{u}^{k}+\theta e^{k}, x, t\right) d \theta
$$

Remark 1: The well-posedness for the system (3) and the error system (8)-(9) can be proven with the same arguments than those used in [7], see also [10]. For instance, consider

$$
w(t)=e^{1}(., t)
$$

of the error system (8). The equation (8) can be rewritten as a differential equation in the Hilbert space $H=L_{2}(0, l)$ with the norm $\|\cdot\|_{L_{2}}$

$$
\dot{w}(t)=A w(t)+F(t, w(t)), \quad t \geq 0
$$

where the operator $A$ is defined by:

$$
A=\frac{\partial^{2}}{\partial x^{2}}
$$

and has the dense domain:

$$
\mathcal{D}_{2}(A)=\left\{w \in \mathcal{H}^{2}(0, l): w(0)=w(l)=0\right\} .
$$

The nonlinear term $F: R \times \mathcal{H}^{2}(0, l) \rightarrow L_{2}(0, l)$ is defined on functions $w(., t)$ as:

$$
\begin{aligned}
F(t, w(., t))= & \phi(x, t, w(x, t)) w(x, t)-L w\left(\bar{x}_{j}, t-\frac{D}{m}\right) \\
= & \phi(x, t, w(x, t)) w(x, t)-L w\left(x, t-\frac{D}{m}\right) \\
& +L \int_{\bar{x}_{j}}^{x} w_{\xi}\left(\xi, t-\frac{D}{m}\right) d \xi d x
\end{aligned}
$$

Let us also define the following Hilbert space

$$
\mathcal{D}_{1}(A)=\left\{w \in \mathcal{H}^{1}(0, l): w(0)=w(l)=0\right\},
$$

Using relevant material on fractional operators degrees [11] and Henry's theorem [12], we get: if $w\left(t_{0}\right) \in \mathcal{D}_{1}(A)$, then

$$
w(t)-w\left(t_{0}\right)=\int_{t_{0}}^{t}[A w(s)+F(s, w(s))] d s
$$

holds for all $t \geq t_{0}$.

Theorem 1: Given $D$ and $m$, consider the system (3) and the observer (4)-(5). Given positive constants $\Delta, \delta, L>M_{f}-$ $\frac{\pi^{2}}{l^{2}}, R$ and $\delta_{1}$ such that $2 \delta>\delta_{1}$, let there exist positive scalars $p_{1}, p_{2}, p_{3}, r$ and $g$ such that :

$$
\delta p_{3}<p_{2} ; \frac{\Delta}{\pi} L R^{-1}\left(p_{3}+p_{2}\right)<\delta_{1} p_{3}
$$

and

$$
\Phi_{m_{f}}<0 ; \Phi_{M_{f}}<0
$$

where

$$
\Phi_{\phi}=\left(\begin{array}{ccc}
\Phi_{11}-\lambda & \Phi_{12} & \Phi_{13} \\
\Phi_{12} & \Phi_{22} & \Phi_{23} \\
\Phi_{13} & \Phi_{23} & \Phi_{33}
\end{array}\right)
$$

with

$$
\begin{aligned}
\Phi_{11} & =2 \delta p_{1}+g-r e^{-2 \delta \frac{D}{m}}+2 p_{2}\left(\phi+\frac{\Delta}{2 \pi} L R\right) \\
\Phi_{12} & =-p_{2}+p_{1}+p_{3} \phi \\
\Phi_{13} & =r e^{-2 \delta \frac{D}{m}}-p_{2} L \\
\Phi_{22} & =\frac{\Delta L R p_{3}}{\pi}-2 p_{3}+r\left(\frac{D}{m}\right)^{2} \\
\Phi_{23} & =-L p_{3} \\
\Phi_{33} & =-(r+g) e^{-2 \delta \frac{D}{m}} \\
\lambda & =\frac{2 \pi^{2}}{l^{2}}\left(p_{2}-\delta p_{3}\right) .
\end{aligned}
$$

Then all the observation errors $\int_{0}^{1}\left(e_{x}^{k}(x, t)\right)^{2} d x$ and $\int_{0}^{1}\left(e_{x}^{k}(x, t)\right)^{2} d x(k=1, . ., m)$ globally exponentially decay to zero as $t \rightarrow+\infty$. The above LMIs are always feasible for large enough $m$.

Proof :

Consider (8) and the corresponding Lyapunov-Krasovskii functional (as in [7]):

$$
\begin{aligned}
V^{1}(t) & =p_{1} \int_{0}^{l}\left(e^{1}(x, t)\right)^{2} d x+p_{3} \int_{0}^{l}\left(e_{x}^{1}(x, t)\right)^{2} d x \\
& +g \int_{0}^{l}\left[\int_{t-\frac{D}{m}}^{t} e^{2 \delta(s-t)}\left(e^{1}(x, s)\right)^{2} d s\right] d x \\
& +\frac{D}{m} r \int_{0}^{l}\left[\int_{-\frac{D}{m}}^{0} \int_{t+\theta}^{t} e^{2 \delta(s-t)}\left(e_{s}^{1}(x, s)\right)^{2} d s d \theta\right] d x
\end{aligned}
$$


Differentiating the above functional we find:

$$
\begin{aligned}
\dot{V}^{1}(t)+2 \delta V^{1}(t) & =2 p_{1} \int_{0}^{l} e^{1}(x, t) e_{t}^{1}(x, t) d x \\
& +2 p_{3} \int_{0}^{l} e_{x}^{1}(x, t) e_{x t}^{1}(x, t) d x \\
& -\frac{D}{m} r \int_{0}^{l} \int_{t-\frac{D}{m}}^{t} e^{2 \delta(s-t)} e_{s}^{1}(x, s)^{2} d s d x \\
& +\int_{0}^{l}\left[( \frac { D } { m } ) ^ { 2 } r \left(e_{t}^{1}(x, t)^{2}+g\left(e^{1}(x, t)\right)^{2}\right.\right. \\
& \left.-g e^{-2 \delta \frac{D}{m}}\left(e^{1}\left(x, t-\frac{D}{m}\right)\right)^{2}\right] d x \\
& +2 \delta p_{1} \int_{0}^{l}\left(e^{1}(x, t)\right)^{2} d x \\
& +2 \delta p_{3} \int_{0}^{l}\left(e_{x}^{1}(x, t)\right)^{2} d x
\end{aligned}
$$

We use further Jensen's inequality:

$$
\begin{aligned}
& -\quad \frac{D}{m} r \int_{0}^{l} \int_{t-\frac{D}{m}}^{t} e^{2 \delta(s-t)} e_{s}^{1}(x, s)^{2} d s d x \leq \\
& -r \quad \int_{0}^{l} e^{-2 \delta \frac{D}{m}}\left(\int_{t-\frac{D}{m}}^{t} e^{2 \delta(s-t)} e_{s}^{1}(x, s) d s\right)^{2} d x
\end{aligned}
$$

and employ th descriptor method [14], where the right-hand side of the following equation is added to $\dot{V}^{1}$ :

$$
\begin{aligned}
0 & =2 \int_{0}^{l}\left[p_{2} e^{1}(x, t)+p_{3} e_{t}^{1}(x, t)\right]\left[-e_{t}^{1}(x, t)+e_{x x}^{1}(x, t)\right. \\
& \left.+\Psi\left(x, t, e^{1}\right) e^{1}(x, t)-L e^{1}\left(x, t-\frac{D}{m}\right)\right] d x \\
& +2 \sum_{j=0}^{N-1} \int_{x_{j}}^{x_{j+1}}\left[p_{2} e^{1}(x, t)+p_{3} e_{t}^{1}(x, t)\right] \\
& \times \quad L \int_{\bar{x}_{j}}^{x} e_{\xi}^{1}\left(\xi, t-\frac{D}{m}\right) d \xi d x .
\end{aligned}
$$

Here $p_{2}$ and $p_{3}$ are free parameters.

From (22)-(24), by using Young and Wirtinger inequalities we arrive at:

$$
\begin{aligned}
\dot{V}^{1}(t) & +2 \delta V^{1}(t) \leq \int_{0}^{l} \eta^{T} \Phi_{\phi} \eta d x \\
& +\frac{\Delta}{\pi} L R^{-1}\left(p_{3}+p_{2}\right) \int_{0}^{l}\left(e_{x}^{1}\left(x, t-\frac{D}{m}\right)\right)^{2} d x
\end{aligned}
$$

where $\eta=\operatorname{col}\left\{e^{1}(x, t), e_{t}^{1}(x, t), e^{1}\left(x, t-\frac{D}{m}\right)\right\}$. Since $\Phi_{\phi}$ is affine in $\phi$, then under (18):

$$
\int_{0}^{l} \eta^{T} \Phi_{\phi} \eta d x \leq 0 .
$$

From this we also deduce

$$
\begin{aligned}
\dot{V}^{1}(t) & +2 \delta V^{1}(t)-\delta_{1} V^{1}\left(t-\frac{D}{m}\right) \leq \int_{0}^{l} \eta^{T} \Phi_{\phi} \eta d x \\
& +\left(\frac{\Delta}{\pi} L R^{-1}\left(p_{3}+p_{2}\right)-\delta_{1}\right) \int_{0}^{l}\left(e_{x}^{1}\left(x, t-\frac{D}{m}\right)\right)^{2} d x
\end{aligned}
$$

Then we conclude under conditions of Theorem 1, that

$$
\dot{V}^{1}(t)+2 \delta V^{1}(t)-\delta_{1} V^{1}\left(t-\frac{D}{m}\right) \leq 0
$$

Consider further the observation error equations (9) with $k=2, \ldots, m$. The only difference between the above system and the one of the case $k=1$ is in the disturbing term $\int_{0}^{\bar{x}_{j}} e_{x}^{k-1}(x, t) d x$. So, under under the strict LMIs (18), the Lyapunov-Krasovskii functional

$$
\begin{aligned}
& V^{k}(t)=p_{1} \int_{0}^{l}\left(e^{k}(x, t)\right)^{2} d x+p_{3} \int_{0}^{l}\left(e_{x}^{k}(x, t)\right)^{2} d x \\
& +g \int_{0}^{l}\left[\int_{t-\frac{D}{m}}^{t} e^{2 \delta(s-t)}\left(e^{k}(x, s)\right)^{2} d s\right] d x \\
& +\frac{D}{m} r \int_{0}^{l}\left[\int_{-\frac{D}{m}}^{0} \int_{t+\theta}^{t} e^{2 \delta(s-t)}\left(e_{s}^{k}(x, s)\right)^{2} d s d \theta\right] d x, \\
& k=2, \ldots, m
\end{aligned}
$$

satisfies the following inequality

$$
\dot{V}^{k}(t)+\left(2 \delta-\epsilon \gamma^{2}\right) V^{k}(t)-\delta_{1} V^{k}\left(t-\frac{D}{m}\right)-\gamma^{2} V^{k-1}(t) \leq 0
$$

along (9), where $\gamma^{2}$ is large enough and $\epsilon>0$ is small enough subject to

$$
2 \delta-\epsilon \gamma^{2}>\delta_{1} .
$$

Similar to [17], consider next the following Lyapunov-Krasovskii functional for the augmented system (8)-(9):

$$
V(t)=\sum_{k=1}^{m} \epsilon^{k-1} V^{k}(t)
$$

Then multiplying (29) by $\epsilon^{k-1}$ and summing with (28) we arrive at

$$
\begin{aligned}
& \dot{V}(t)+\left(2 \delta-\epsilon \gamma^{2}\right) V(t)-\delta_{1} \text { sup }_{-h \leq s \leq 0} V(t+s) \\
& \leq \dot{V}(t)+\left(2 \delta-\epsilon \gamma^{2}\right) V(t)-\delta_{1} V\left(t-\frac{D}{m}\right) \leq 0 .
\end{aligned}
$$

that due to (30) and Halanay's inequality implies the exponential convergence of $V$.

Remark 2: The LMIs in Theorem 1 depend on the fraction $D / m$. If they are feasible for $H_{\max }=D / m$, then choosing $m \geq D / H_{\max }$ we have always a feasible LMI. Then for each delay $D$, we can find a sufficiently large $m$ such that the LMIs of the Theorem 1 are verified.

\section{SAMPLED MEASUREMENTS CASE}

In this section, we present the extension of the above observer to sampled- measurements case. In this case the output is available only at sampling instants $t_{i}$

$$
0=t_{0}<t_{1}<\ldots<t_{i}<\ldots, \quad \lim _{k \rightarrow \infty} t_{i}=\infty .
$$

We assume that the sampling intervals may be variable, but upper-bounded by a known bound $h$ :

$$
t_{i+1}-t_{i} \leq h \quad \forall i=0,1, \ldots
$$


The proposed observer has the following form : for $k=1$ :

$$
\begin{aligned}
\hat{u}_{t}^{1}(x, t)= & \hat{u}_{x x}^{1}(x, t)+f\left(\hat{u}^{1}(x, t), x, t\right) \\
- & L\left(\hat{u}^{1}\left(\bar{x}_{j}, t_{i}-\frac{D}{m}\right)-y\left(t_{i}\right)\right), \\
& \forall t \in\left[t_{i}, t_{i+1}\right), \quad \forall x \in\left[x_{j}, x_{j+1}\right),
\end{aligned}
$$

for $k=2, \ldots, m$ :

$$
\begin{aligned}
\hat{u}_{t}^{k}(x, t)= & \hat{u}_{x x}^{k}(x, t)+f\left(\hat{u}^{k}(x, t), x, t\right) \\
- & L\left(\hat{u}^{k}\left(\bar{x}_{j}, t-\frac{D}{m}\right)-\hat{u}^{k-1}\left(\bar{x}_{j}, t\right)\right), \\
& \forall t \in\left[t_{i}, t_{i+1}\right), \quad \forall x \in\left[x_{j}, x_{j+1}\right),
\end{aligned}
$$

Theorem 2: Given $D, h$ and $m$, consider the system (3) and the observer (32)-(33). Given positive constants scalars $\Delta, \delta, L>M_{f}-\frac{\pi^{2}}{l^{2}}, R$ and $\delta_{1}$ such that $2 \delta>\delta_{1}$, let there exist positive scalars $p_{1}, p_{2}, p_{3}, r, W$ and $g$ such that :

$$
\delta p_{3}<p_{2} ; \frac{\Delta}{\pi} L R^{-1}\left(p_{3}+p_{2}\right)<\delta_{1} p_{3}
$$

and

$$
\Phi_{m_{f}}<0 \quad \Phi_{M_{f}}<0
$$

where

$$
\Phi_{\phi}=\left(\begin{array}{cccc}
\Phi_{11}-\lambda & \Phi_{12} & \Phi_{13} & p_{2} L \\
\Phi_{12} & \Phi_{22}+W h^{2} e^{2 \delta h} & \Phi_{23} & p_{3} L \\
\Phi_{13} & \Phi_{23} & \Phi_{33} & 0 \\
p_{2} L & p_{3} L & 0 & -W \frac{\pi^{2}}{4}
\end{array}\right)
$$

with $\Phi_{11}, \Phi_{12}, \Phi_{13}, \Phi_{22}, \Phi_{23}$ and $\Phi_{33}$ given by (20). Then all the observation errors $\int_{0}^{1}\left(e^{k}(x, t)\right)^{2} d x$ and $\int_{0}^{1}\left(e_{x}^{k}(x, t)\right)^{2} d x(k=1, \ldots, m)$ globally exponentially decay to zero as $t \rightarrow+\infty$.

Proof : The observation error is described by the following equations :

for $k=1$ :

$$
\begin{aligned}
e_{t}^{1}(x, t)= & e_{x x}^{1}(x, t)+\phi\left(x, t, e^{1}\right)\left(\hat{u}^{1}(x, t)-u^{1}(x, t)\right) \\
- & L e^{1}\left(\bar{x}_{j}, t_{i}-\frac{D}{m}\right), \\
& \forall t \in\left[t_{i}, t_{i+1}\right), \quad \forall x \in\left[x_{j}, x_{j+1}\right) \\
e^{1}(l, t)= & e^{1}(0, t)=0,
\end{aligned}
$$

for $k=2, \ldots, m$ :

$$
\begin{aligned}
e_{t}^{k}(x, t)= & e_{x x}^{k}(x, t)+\phi\left(x, t, e^{k}\right)\left(\hat{u}^{k}(x, t)-u^{k}(x, t)\right) \\
- & L e^{k}\left(\bar{x}_{j}, t-\frac{D}{m}\right)+L e^{k-1}\left(\bar{x}_{j}, t\right), \\
& \forall t \in\left[t_{i}, t_{i+1}\right), \quad \forall x \in\left[x_{j}, x_{j+1}\right), \\
e^{k}(l, t)= & e^{k}(0, t)=0,
\end{aligned}
$$

As we can easily see, the unique difference with the observer without sampling measurements is for the first sub-observer $(k=1)$. In order to study the convergence of the case $k=1$, we use the following modified Lyapunov-Krasvoskii inspired from [8], [15]:

$$
\begin{aligned}
& V_{W}^{1}(t)=V^{1}(t)+W^{1}(t), \\
& W^{1}(t)=W h^{2} e^{2 \delta h} \int_{0}^{l} \int_{t_{i}-\frac{D}{m}}^{t} e^{2 \delta(s-t)}\left(e_{s}^{1}(x, s)\right)^{2} d s d x \\
& -\frac{\pi^{2}}{4} W \int_{0}^{l} \int_{t_{i}-\frac{D}{m}}^{t-\frac{D}{m}} e^{2 \delta(s-t)}\left[e^{1}(x, s)-e^{1}\left(x, t_{i}-\frac{D}{m}\right)\right]^{2} d s d x .
\end{aligned}
$$

By generalized Wirtinger's inequality [15], we deduce $W^{1}$ is nonnegative and does not grow in the jumps [15]. Moreover,

$$
\begin{aligned}
& \dot{W}^{1}(t)+2 \delta W^{1}(t) \leq h^{2} e^{2 \delta h} \int_{0}^{l}\left(e_{t}^{1}(x, s)\right)^{2} d x \\
& -\frac{\pi^{2}}{4} \int_{0}^{l}\left[e^{1}\left(x, t-\frac{D}{m}\right)-e^{1}\left(x, t_{i}-\frac{D}{m}\right)\right]^{2} d x .
\end{aligned}
$$

Adding the latter inequality to (28) we conclude that under conditions of Theorem $2 \forall t \in\left[t_{i}, t_{i+1}\right)$ the following inequality holds:

$$
\dot{V}_{W}^{1}(t)+2 \delta V_{W}^{1}(t)-\delta_{1} V_{W}^{1}\left(t_{i}-\frac{D}{m}\right) \leq 0
$$

For $k \geq 2$ the observation error is described by the following equation :

$$
\begin{aligned}
e_{t}^{k}(x, t)= & e_{x x}^{k}(x, t)+\phi\left(x, t, e^{k}\right) e^{k}(x, t) \\
- & L e^{k}\left(\bar{x}_{j}, t-\frac{D}{m}\right)+L \int_{0}^{\bar{x}_{j}} e_{x}^{k-1}(x, t) d x, \\
& \forall x \in\left[x_{j}, x_{j+1}\right), \\
e^{k}(l, t)= & e^{k}(0, t)=0,
\end{aligned}
$$

As we can see the systems (41) and (9) are identical. Then, the exponential convergence of the observer (32)-(33) can be proved by using arguments of Theorem 1 with $V^{1}$ changed by $V_{W}^{1}$.

\section{NUMERICAL ILLUSTRATION}

Let us consider the following example :

$$
u_{t}=u_{x x}(x, t)+1.02 \pi^{2} u(x, t)
$$

with $u(x, 0)=\sin (x)$ and let $y_{j}\left(t_{k}\right)=u\left(\bar{x}_{j}, t_{k}-D\right), j=$ $1, \ldots, N-1$, where $D$ is an arbitrarily delay and $\hat{u}(x, 0)=0$. It has to be noticed that the above system is unstable.

We choose $L=1, \Delta=\frac{1}{50}, \delta=0.21$ and $\delta_{1}=0.1, l=$ $1, R=1$.

By solving LMIs of Theorem 2, we deduce the following table which illustrates the maximum value of the ratio $D / m$ for different values of $h$.

\begin{tabular}{|c|llll|}
\hline$h$ & 0.1 & 0.25 & 0.5 & 0.57 \\
\hline$\frac{D}{m}$ & 0.37 & 0.28 & 0.11 & 0.056 \\
\hline
\end{tabular}

The simulations (Fig1-4) show clearly that the real values of the number $(m)$ of required sub-observers in the chain is less conservative than the one provided by LMIs.

For instance, in (Fig 1), we can see that for $D=0.5 \mathrm{~s}$ and $h=0.5 s$ only $m=1$ observer is required to ensure exponential convergence whereas the LMIs indicate that $m=5$. This result confirms the fact that the LMIs are only sufficient conditions. 

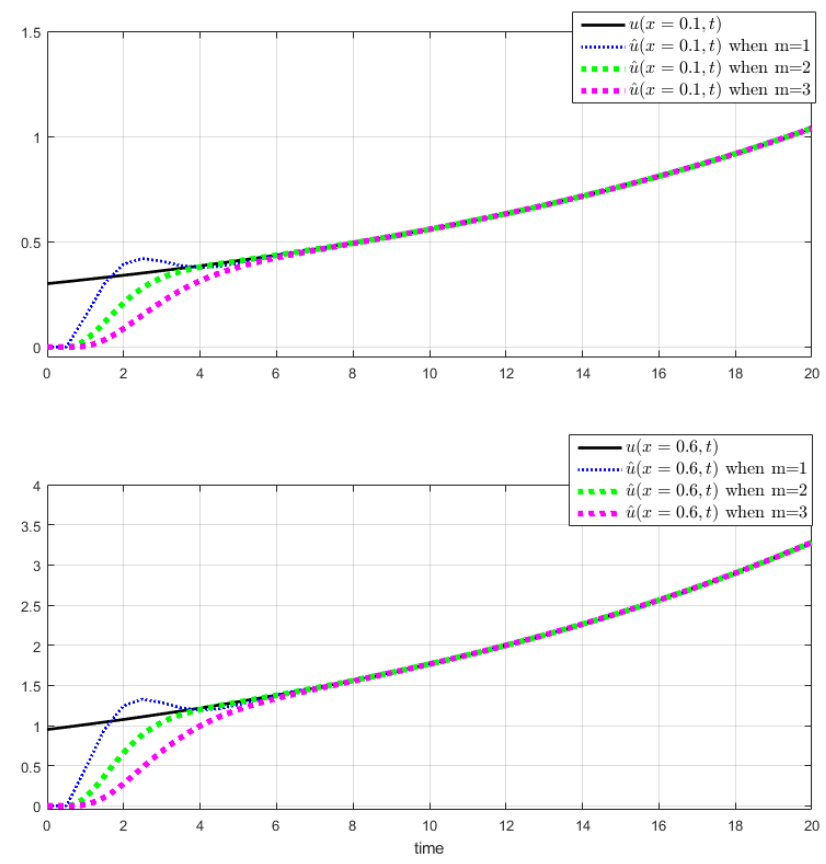

Figure 1. The state $u(x, t)$ and its observations for $m=1$ to $m=3$ at $x=0.1$ and $x=0.6$ for a delay $D=0.5$ s and sampling period $h=0.5 \mathrm{~s}$.
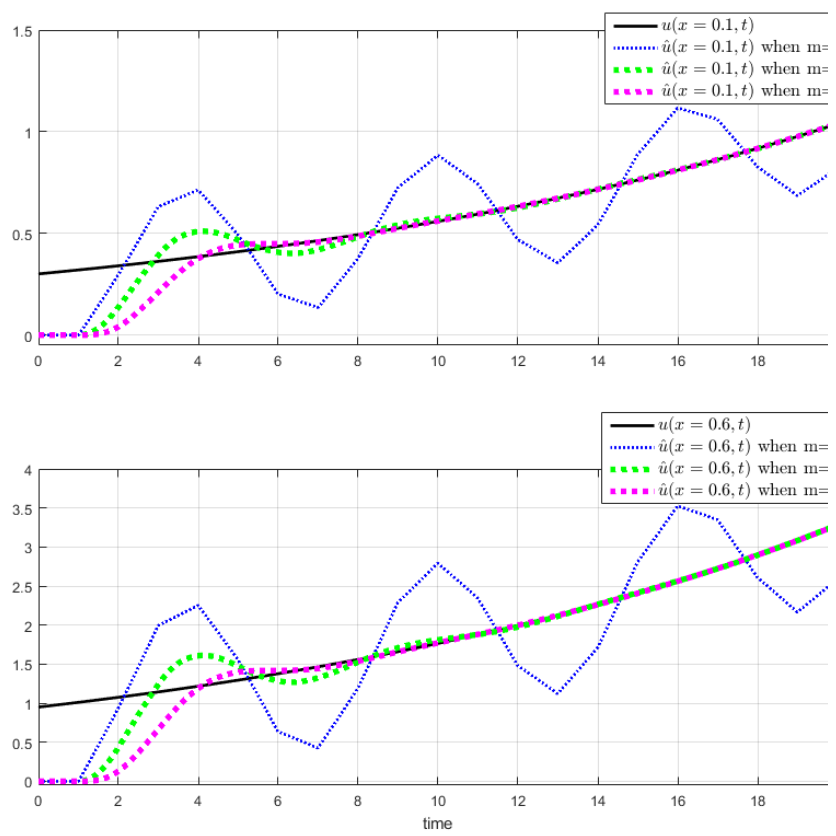

Figure 2. The state $u(x, t)$ and its observations for $m=1$ to $m=3$ at $x=0.1$ and $x=0.6$ for a delay $D=1$ s and sampling period $h=1$ s.
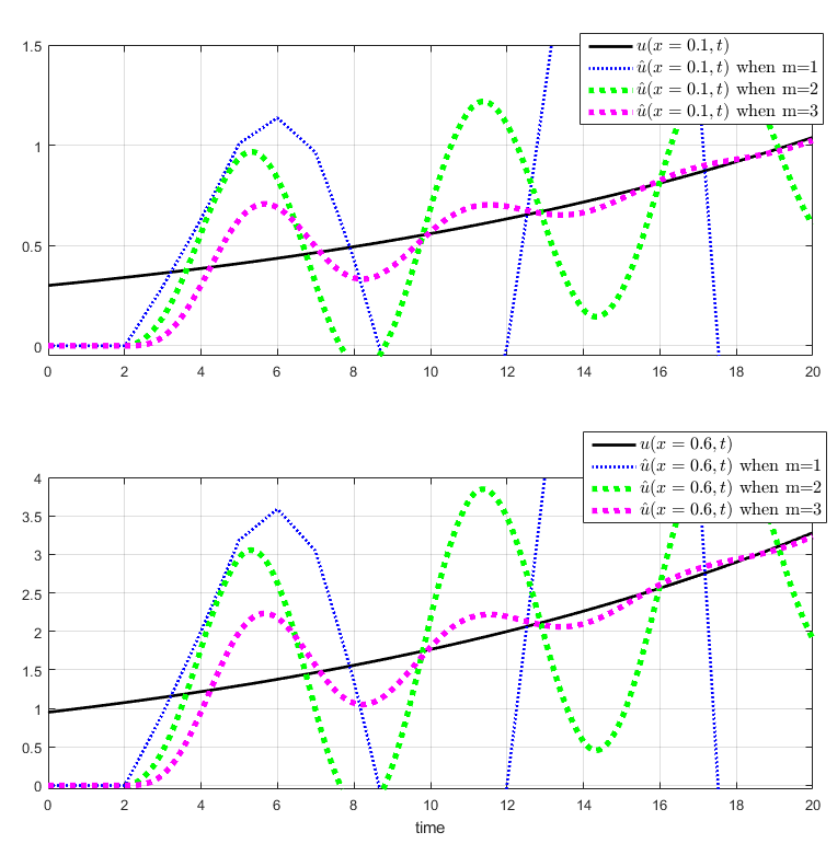

Figure 3. The state $u(x, t)$ and its observations for $m=1$ to $m=3$ at $x=0.1$ and $x=0.6$ for a delay $D=2$ s and sampling period $h=1 \mathrm{~s}$.
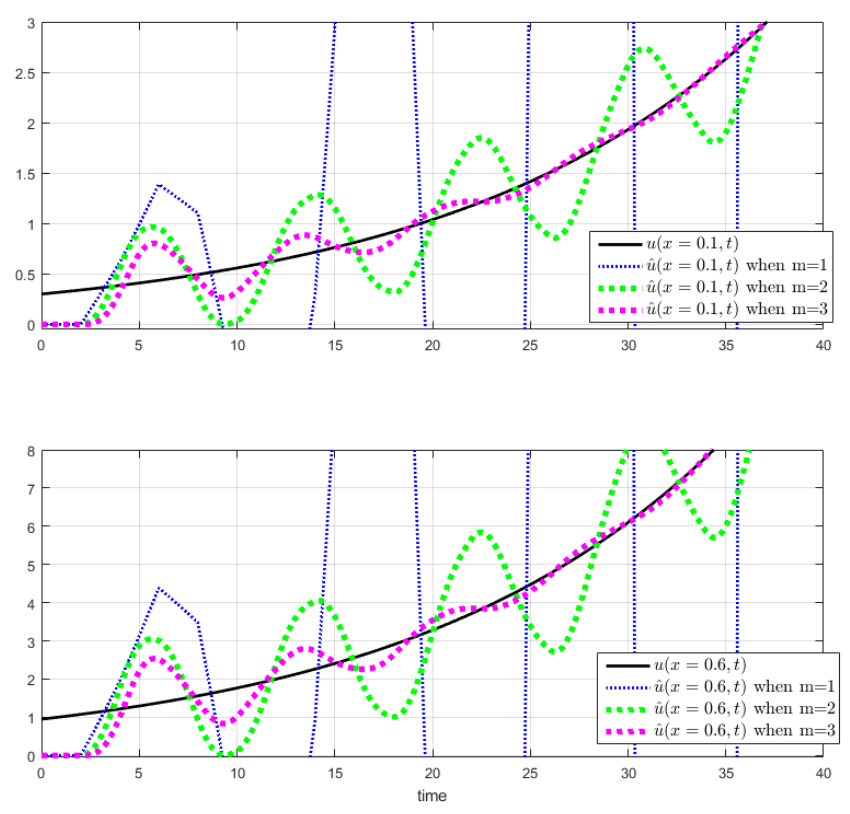

Figure 4. The state $u(x, t)$ and its observations for $m=1$ to $m=3$ at $x=0.1$ and $x=0.6$ for a delay $D=2 \mathrm{~s}$ and sampling period $h=2 \mathrm{~s}$. 


\section{CONCLUSION}

In this paper, a novel observer is proposed for a class of parabolic systems with delayed and sampled measurements. The main advantage provided by this algorithm is that it can handle arbitrary delay with a sufficiently small maximum allowable sampling period. It has to be noticed that this method can be extended to several important classes of infinite-dimensional systems. Our main challenge in the future is the extension to adaptive observers case.

\section{REFERENCES}

[1] F. Cacace, A. Germani, and C. Manes, " Nonlinear systems with multiple time-varying measurement delays," SIAM J. Control Optim., vol. 52 no. 3, pp. 1862-1885, 2014.

[2] A. Germani, C. Manes, and P. Pepe, "A new approach to state observation of nonlinear systems with delayed output," IEEE Transactions on Automatic Control, vol. 47, no. 1, pp. 96-101, 2002.

[3] T. Ahmed-Ali, E. Cherrier, and F. Lamnabhi-Lagarrigue, "Cascade high predictors for a class of nonlinear systems," IEEE Trans. on Aut. Control, vol. 57, pp. 224-229, 2012.

[4] G. Besançon, D. Georges, and Z. Benayache, (2007). "Asymptotic state prediction for contiunous-time systems with delayed input and application to control," Presented at The Proceedings of the European Control Conference, pp. 2-5.

[5] M. Krstic. "Delay Compensation for Nonlinear, Adaptive, and PDE Systems." Basel: Birkhuser, 2009.

[6] T. Ahmed-Ali, F. Giri, M. Krstic, M. Kahelras, "PDE based observer design for nonlinear systems with large output delay," Systems \& Control Letters, vol. 113, pp.1-8, 2018.

[7] E. Fridman, and A. Blighovsky, "Robust sampled-data control of a class of semilinear parabolic systems," Automatica, vol. 48, pp. 826-836, 2012 .

[8] K. Liu, and E. Fridman, "Wirtinger inequality and lyapunov-based sampled-data stabilization," Automatica, vol. 48, pp. 102-108, 2012.

[9] A. Halanay, "Differential Equations: Stability, Oscillations, Time Lags," Academic Press, New York. 1966

[10] T. Ahmed-Ali, E. Fridman, F. Giri, L. Burlion, and F. Lamnabhi-Lagarrigue, "Using exponential time-varying gains for sampled-data stabilization and estimation," Automatica, vol. 67, pp. 244-251, 2016.

[11] M. Tucsnak, and G. Weiss, "Observation and control for operator semigroups". Basel: Birkhauser. 2009

[12] D. Henry, "Geometric theory of semilinear parabolic equations," New York: Springer-Verlag, 1993.

[13] W. Kang, and E. Fridman, "Boundary Constrained Control of Delayed Nonlinear Schrodinger Equation," IEEE Trans. Automatic Control, vol. 63, no. 11 , pp. 3873-3880, 2018

[14] E. Fridman, "Systems and Control: Foundations and Applications, Introduction to Time-Delay Systems: Analysis and Control." Basel: Birkhauser, 2014.

[15] A. Selivanov, and E. Fridman, "Observer-based input-to-state stabilization of networked control systems with large uncertain delays." Automatica , vol. 74, pp. 63-70, 2016.

[16] T. Ahmed-Ali, E. Fridman, F. Giri, M. Kahelras, F. Lamnabhi-Lagarrigue, and L. Burlion, "Observer design for a class of parabolic systems with arbitrarily delay measurements Observer-based input-to-state stabilization of networked control systems with large uncertain delays. To Appear IEEE CDC. 2018

[17] E., Fridman, C. Bonnet, F. Mazenc, and W. Djema, "Stability of the cell dynamics in Acute Myeloid Leukemia." Systems \& Control Letters, vol. 88, pp. 91-100, 2016. 